TI 2011-063/4

Tinbergen Institute Discussion Paper

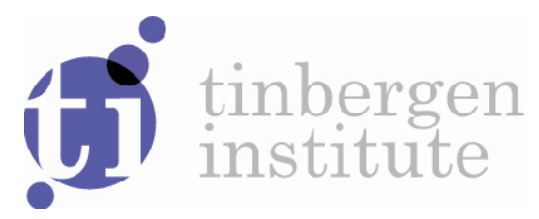

\title{
Forecasting the U.S. Term Structure of Interest Rates using a Macroeconomic Smooth Dynamic Factor Model
}

Siem Jan Koopmana

Michel van der Welb

a VU University Amsterdam, and Tinbergen Institute;

${ }^{b}$ Erasmus School of Economics, ERIM Rotterdam, and CREATES, Aarhus. 
Tinbergen Institute is the graduate school and research institute in economics of Erasmus University Rotterdam, the University of Amsterdam and VU University Amsterdam.

More TI discussion papers can be downloaded at http://www.tinbergen.nl

Tinbergen Institute has two locations:

Tinbergen Institute Amsterdam

Gustav Mahlerplein 117

1082 MS Amsterdam

The Netherlands

Tel.: +31(0)205251600

Tinbergen Institute Rotterdam

Burg. Oudlaan 50

3062 PA Rotterdam

The Netherlands

Tel.: +31(0)10 4088900

Fax: +31(0)104089031

Duisenberg school of finance is a collaboration of the Dutch financial sector and universities, with the ambition to support innovative research and offer top quality academic education in core areas of finance.

DSF research papers can be downloaded at: http://www.dsf.nl/

Duisenberg school of finance

Gustav Mahlerplein 117

1082 MS Amsterdam

The Netherlands

Tel.: +31(0)20 5258579 


\title{
Forecasting the U.S. Term Structure of Interest Rates using a Macroeconomic Smooth Dynamic Factor Model
}

\author{
Siem Jan Koopman ${ }^{(a, b)}$ Michel van der Wel ${ }^{(b, c)}$ \\ (a) Department of Econometrics, VU University Amsterdam \\ (b) Tinbergen Institute \\ (c) Erasmus School of Economics, ERIM Rotterdam and CREATES, Aarhus
}

April 6, 2011

Some keywords: Fama-Bliss data set; Kalman filter; Maximum likelihood; Yield curve.

JEL classification: C32, C51, E43.

Acknowledgements: We would like to thank Dick van Dijk and Peter Exterkate for providing the macroeconomic dataset to us. Further details of our estimation procedure and the results reported in this paper are available from the authors upon request. Michel van der Wel acknowledges the support from CREATES, funded by the Danish National Research Foundation.

Address of correspondence: S.J. Koopman, Department of Econometrics, VU University Amsterdam, De Boelelaan 1105, NL-1081 HV Amsterdam, The Netherlands.

Emails: s.j.koopman@feweb.vu.nl vanderwel@ese.eur.nl 


\title{
Forecasting the U.S. Term Structure of Interest Rates using a Macroeconomic Smooth Dynamic Factor Model
}

\author{
Siem Jan Koopman and Michel van der Wel
}

\begin{abstract}
We extend the class of dynamic factor yield curve models for the inclusion of macroeconomic factors. We benefit from recent developments in the dynamic factor literature for extracting the common factors from a large panel of macroeconomic series and for estimating the parameters in the model. We include these factors into a dynamic factor model for the yield curve, in which we model the salient structure of the yield curve by imposing smoothness restrictions on the yield factor loadings via cubic spline functions. We carry out a likelihood-based analysis in which we jointly consider a factor model for the yield curve, a factor model for the macroeconomic series, and their dynamic interactions with the latent dynamic factors. We illustrate the methodology by forecasting the U.S. term structure of interest rates. For this empirical study we use a monthly time series panel of unsmoothed Fama-Bliss zero yields for treasuries of different maturities between 1970 and 2009, which we combine with a macro panel of 110 series over the same sample period. We show that the relation between the macroeconomic factors and yield curve data has an intuitive interpretation, and that there is interdependence between the yield and macroeconomic factors. Finally, we perform an extensive out-of-sample forecasting study. Our main conclusion is that macroeconomic variables can lead to more accurate yield curve forecasts.
\end{abstract}




\section{Introduction}

Much interest has been given to the forecasting of interest rates for different maturities, known as the yield curve or the term structure of interest rates. Initially, forecasting has been based only on yield curve information. Recent literature has shown the strong relation between the yield curve and macroeconomic developments; see, for example, Ang and Piazzesi (2003) and Diebold, Rudebusch, and Aruoba (2006). It has given a renewed focus on using macroeconomic information in forecasting the yield curve; see, for example, Moench (2008) and Exterkate, Van Dijk, Heij, and Groenen (2010). We construct a dynamic factor model for a joint likelihood-based analysis of the term structure of interest rates and a large panel of macroeconomic series. In particular, estimating parameters, extracting the factors from the joint dynamic factor model and forecasting the term structure of interest rates are the aims of our analysis.

In our analysis we benefit from contributions to the literature on the general dynamic factor model, which increasingly plays a major role in econometrics. Early contributions to this literature can be found in Sargent and Sims (1977), Geweke (1977), Geweke and Singleton (1981), Engle and Watson (1981), Watson and Engle (1983), Connor and Korajczyk (1993) and Gregory, Head, and Raynauld (1997). Most of these papers consider time series panels with limited panel dimensions. The increasing availability of high dimensional data sets has intensified the quest for computationally efficient estimation methods. The strand of literature headed by Forni, Hallin, Lippi, and Reichlin (2000), Stock and Watson (2002) and Bai (2003) led to a renewed interest in dynamic factor analysis. These methods are typically applied to high dimensional panels of time series. Exact maximum likelihood methods such as proposed in Watson and Engle (1983) have traditionally been dismissed as computationally too intensive for such high dimensional panels. Exceptions are studies such as Quah and Sargent (1993) and Doz, Giannone, and Reichlin (2010) who consider moderately sized panels of economic time series in their studies. Jungbacker and Koopman (2008) however present new results that facilitate application of exact maximum likelihood methods for very high dimensional panels.

In this paper we employ an econometric likelihood-based framework based on a dynamic factor model with smooth factor loadings as considered by Jungbacker, Koopman, and van der Wel (2010). The smoothness conditions on the loadings are introduced via spline functions that depend on knot coefficients, see Poirier (1976). The parameters in the model are estimated via a maximum likelihood procedure that allows for imposing smoothness in the factor structure. There are several motivations to impose smoothness on the factor loadings in a dynamic factor model. The economic motivation is to establish an inter- 
pretation for the factors. When the factor loadings are related to particular characteristics of the corresponding variables in the panel, we can impose this relationship by specifying a smooth flexible function for the factor loading coefficients. A smooth pattern in a column of the loading matrix can lead to an interpretable factor that is associated with this column. In our empirical study for a panel of interest rates, we impose smoothness on the loadings through a spline function that depends on time to maturity. The common interpretation of the factors as level, slope and curvature of the yield curve can be established. Also for the macroeconomic variables we can consider a smooth relationship between the underlying factors and observations. The econometric motivation of smooth loadings is partly the aim for a parsimonious model specification where individual loading coefficients are interpolated by a flexible function that depends on a small number of coefficients. The precision of parameter estimates is generally increased by considering more parsimonious models. Furthermore, smoothness in factor loadings may also lead to models that are more robust to aberrant observations. It is also often argued that forecasts based on a model with a small set of parameters can be expected to be more precise than those based on a less parsimonious model; see the discussion in Clements and Hendry (1998).

We extract a small number of latent factors from a large panel of macroeconomic series using the general dynamic factor model. We follow Stock and Watson (2002) who extract latent factors from a similarly large panel of economic time series to forecast eight macroeconomic series. We adopt the Jungbacker and Koopman (2008) likelihood-based framework to extract the common factors from the macro panel. The macro factors are linked with the smooth dynamic factors of the yield curve. In this way we obtain a macroeconomic smooth dynamic factor model that can simultaneously analyse the yields and the panel of macroeconomic series. Estimation and forecasting take place as parts of a unified likelihood-based analysis.

We empirically investigate whether the inclusion of macroeconomic factors in our smooth dynamic factor model is effective by means of a forecasting study for a panel of U.S. interest rate series for different times to maturity. Our primary aim is to investigate whether adding a panel of macroeconomic time series variables can lead to more precise forecasts. The following two alternative model specifications are considered for yield curve forecasting: the dynamic Nelson-Siegel model and the functional signal plus noise model. The first model for the term structure is based on the seminal paper of Nelson and Siegel (1987) in which the yield curve is approximated by a weighted sum of three smooth functions. The form of these three functions depends on a single parameter. Diebold and Li (2006) use the NelsonSiegel framework to develop a two-step procedure for the forecasting of future yields. They show that forecasts obtained from this procedure are competitive with forecasts obtained 
from other standard prediction methods. Diebold, Rudebusch, and Aruoba (2006) integrate the two-step approach into a single dynamic factor model by specifying the Nelson-Siegel weights as an unobserved vector autoregressive process. Koopman, Mallee, and Van der Wel (2010) consider a generalization of the state space approach by allowing for a time-varying parameter that governs the shape of the Nelson-Siegel function and by including conditional heteroskedasticity for the innovations in the model. Due to its popularity amongst practitioners, central bankers and academic researchers, the Nelson-Siegel model serves as our benchmark term structure model. The dynamic Nelson-Siegel model can also be regarded as a special case of the dynamic factor model and we compare it with our smooth dynamic factor model in the empirical study. The second alternative model is recently discussed by Bowsher and Meeks (2008) and represents the term structure as a cubic spline function that is observed with measurement noise. The parameters controlling the shape of the spline are time-varying and modelled as a cointegrated vector autoregressive process with different numbers of lags. We consider a basic version of this model and compare it with the other models in our empirical study that focuses both on in-sample and out-of-sample results.

Our empirical study is based on a newly constructed monthly time series panel of unsmoothed Fama-Bliss zero yields for U.S. treasuries of different maturities between 1970 and 2009. The data set is used to empirically validate the aforementioned models. From the smooth dynamic factor model we obtain the usual level, slope and curvature factors. The panel of 110 macroeconomic series provides factor estimates corresponding to the real economy, price indices, and labor and housing market conditions. Our joint framework yields latent factors which generally carry a similar interpretation as the individual factor models, but highlights interactions between the yield and macro factors and shows a large correlation between the yield series and macro factors. Our main empirical finding is that the macroeconomic smooth dynamic factor model produces forecasts that are more accurate than those for the model without macroeconomic variables, for most maturities and for most forecasting horizons. When we compare our forecasts with those of the dynamic Nelson-Siegel and the functional plus signal models, the accuracy of our forecasts are generally higher.

The structure of the paper is as follows. The macroeconomic smooth dynamic factor model is presented and discussed in section 2. In this section we discuss the methodology to construct dynamic factor models with and without smooth factor loadings, and how to incorporate a high-dimensional panel of macroeconomic time series variables. Section 3 presents and discusses the results of our empirical forecasting study for the U.S. term structure of interest rates with many economic variables. Section 4 concludes and provides suggestions for future research. 


\section{The macroeconomic smooth dynamic factor model}

We introduce the macroeconomic smooth dynamic factor model in four steps. First, section 2.1 discusses the general set-up of the dynamic factor model. Section 2.2 discusses the dynamic factor model with smooth loadings, which is labelled as the smooth dynamic factor model and was introduced by Jungbacker, Koopman, and van der Wel (2010). Section 2.3 introduces the macroeconomic smooth dynamic factor model which adds macroeconomic factors from a large panel of macroeconomic factors to the smooth dynamic factor model. Finally, section 2.4 turns to parameter estimation and signal extraction for our class of dynamic factor models.

\subsection{The dynamic factor model}

We consider a time series panel of $N$ variables with the observation at time $t$ given by the $N \times 1$ vector

$$
y_{t}=\left(y_{1 t}, \ldots, y_{N t}\right)^{\prime}, \quad t=1, \ldots, n
$$

where $y_{i t}$ is the observation for the $i$ th variable in the panel, at time $t$. The vector of all observations in the panel is denoted by $y=\left(y_{1}^{\prime}, \ldots, y_{n}^{\prime}\right)^{\prime}$. The general dynamic factor model is given by

$$
y_{t}=\mu_{y}+\Lambda f_{t}+\varepsilon_{t}, \quad \varepsilon_{t} \sim \operatorname{NID}(0, H), \quad t=1, \ldots, n,
$$

where $\mu_{y}$ is an $N \times 1$ vector of constants, $\Lambda$ is the $N \times r$ factor loading matrix, $f_{t}$ is an $r$-dimensional dynamic stochastic process, $\varepsilon_{t}$ is the $N \times 1$ disturbance vector and $H$ is the $N \times N$ disturbance variance matrix. The Gaussian disturbance vector series $\varepsilon_{t}$ is serially uncorrelated as NID refers to normally and independently distributed. We further assume that the variance matrix of the observation disturbances $H$ is diagonal. It implies that the covariance between the variables in $y_{t}$ depends solely on the latent factor $f_{t}$. The factor $f_{t}$ is treated as a signal generated from a linear dynamic process and it can be specified as

$$
f_{t}=U \alpha_{t}
$$

where the fixed $r \times p$ matrix $U$ relates $f_{t}$ with the $p$-dimensional unobserved state vector $\alpha_{t}$ which is modelled by the dynamic stochastic process

$$
\alpha_{t+1}=\mu_{\alpha}+T \alpha_{t}+R \eta_{t}, \quad \eta_{t} \sim \operatorname{NID}(0, Q), \quad t=1, \ldots, n,
$$


with $p \times 1$ vector of constants $\mu_{\alpha}, p \times p$ transition matrix $T$ and $p \times q$ selection matrix $R$ (consists typically of ones and zeros). The $q \times 1$ disturbance vector $\eta_{t}$ has $q \times q$ variance matrix $Q$ and is uncorrelated with $\varepsilon_{s}$ for all $s, t=1, \ldots, n$. Although dimensions $N, p, q$ and $r$ can be chosen freely, here we consider models which typically have $r \leq p, p \geq q$ and $N>>r$. The vectors $\mu_{y}$ and $\mu_{\alpha}$ and the matrices $\Lambda, H, U, T$ and $Q$ are referred to as system matrices. The general dynamic factor model $(1)-(3)$ can be regarded as a specific case of a linear Gaussian state space model. Its statistical treatment is based on the Kalman filter and maximum likelihood in which the initial state conditions are treated properly; see, among others, Durbin and Koopman (2001). The typical dynamic specification for $f_{t}$ is the vector autoregressive process which can be represented in the form of (2)-(3); see, for example, Box, Jenkins, and Reinsel (1994, Section 14.2). The inclusion of lagged factors in the observation equation (1) can also be established in this form.

The elements of the system matrices may depend on unknown parameters that need to be estimated. To ensure identification we need to impose restrictions on the parameters in the mean vectors $\mu_{y}$ and $\mu_{\alpha}$ together with those in $\Lambda, T$ and $Q$ that govern the covariance structure of the model $(1)-(3)$. We set $\mu_{\alpha}=0$ and estimate $\mu_{y}$ as this is the more general specification. Restrictions on $\Lambda$ are needed because only its column space can be identified uniquely. Several restrictions on $\Lambda$ can be considered. For example, we can impose the normalizing restriction $\Lambda^{\prime} \Lambda=I_{r}$ or we can select $r$ rows of $\Lambda$ and set these equal to subsequent rows of the $r \times r$ identity matrix $I_{r}$. When the first $r$ rows are set equal to $I_{r}$, we interpret the elements of $f_{t}$ as being the first three variables in $y_{t}$ subject to observation noise in $\varepsilon_{t}$. Such restrictions for $\Lambda$ allow us to leave the parameters in $T$ and $Q$ unrestricted. Alternatively, one can set $Q$ equal to the unity factor and impose $r(r-1) / 2$ restrictions in $\Lambda$ (for example, by setting the top right triangle of the matrix to zero). Many other restrictions can be considered as well.

\subsection{The smooth dynamic factor model}

The main assumption of the Jungbacker, Koopman, and van der Wel (2010) version of the smooth dynamic factor model is that the loading coefficients in $\Lambda$ of the dynamic factor model (1) are subject to smoothing restrictions. It is assumed that the $j$ th column of $\Lambda$ can be represented by a smooth interpolating function. Different smoothness functions can be considered. Many classes of interpolating functions rely on a selection of knots in the range of some variable $z$ that is associated with the vector variable $y_{t}$. Then, the scalar $z_{i}$ represents a particular characteristic of the $i$ th variable in $y_{t}$, for $i=1, \ldots, N$. For example, $z_{i}$ can be a measure of size, location or maturity associated with variable $y_{i t}$. We can enforce 
the restrictions that the same loading coefficients for variables with $z_{i}$ in a particular range of values; for example, small, medium and large sizes, when $z_{i}$ represents the size of the $i$ th variable. Alternatively, we can linearly interpolate the loading coefficient between, say, three knot values that are placed at the smallest possible $z$-value (small size), an intermediate $z$ value (medium size) and the largest possible $z$-value (large size). In both case we reduce the estimation of $N$ coefficients in a column of $\Lambda$ to a small number of coefficients that equals the number of groups or the number of knots (in the example, three). For the interpolation of the loading coefficients in each column of $\Lambda$, we adopt the cubic spline function as discussed by Poirier (1976) and Monahan (2001). The cubic spline function is a third-order polynomial between the knots and it is twice continuously differentiable at the knots. We assume that for each column in $\Lambda$, a $z$ variable is selected and its values are known or observed for each $i$ th variable in $y_{t}$, with $i=1, \ldots, N$. The $z$ variable can be different for different columns of $\Lambda$. We also assume that the variable $x_{i}$ does not change with time-index $t$ although this assumption is not necessary for the implementation of our method. The number and location of knots for the $z$ variable determine the smoothness of the spline function. In our empirical study of section 3 , we have $y_{i t}$ as the interest rate of an U.S. bond and $z_{i}$ as the time to maturity of the bond, for all columns in $\Lambda$.

For our smooth loading functions, we consider the cubic spline representation of Poirier (1976). In our case, it allows expressing the loading coefficients as linear functions of a small set of knot coefficients. For the $j$ th column of $\Lambda$, we assume that a particular $z$ variable is chosen and that the number of knots is set to $k_{j}$. The variable $z_{i}$ characterizes the $i$ th variable in $y_{t}$. For example, it can be age, distance or size. Different columns of $\Lambda$ can adopt a different variable $z$ but it is not necessary. The cubic spline interpolation for the coefficients in the $j$ th column of $\Lambda$ is then given by

$$
\Lambda_{i j}=w_{i j}^{\prime} \bar{\lambda}_{j}, \quad w_{i j}=w\left(z_{i}, \bar{z}_{1}, \ldots, \bar{z}_{k_{j}}\right)
$$

where $\Lambda_{i j}$ is the $(i, j)$ element of loading matrix $\Lambda$ in (1), the $k_{j} \times 1$ vector $w_{i j}$ contains the spline weights and the $k_{j} \times 1$ vector of knot coefficients $\bar{\lambda}_{j}$ are associated with the knot positions $\bar{z}_{1}, \ldots, \bar{z}_{k_{j}}$. The spline weights in vector $w_{i j}$ are determined by the actual value of $z_{i}$ and the $k_{j}$ knot positions. The spline weight function $w()$ is defined through the restrictions associated with the cubic spline, being a third-order polynomial and being twice continuously differentiable at the knots; see Monahan (2001, Chapter 7). When $z_{i}=\bar{z}_{m}$, the weight vector $w_{i}$ is typically equal to the $m$ th column of the identity matrix $I_{k_{j}}$, for any $m=1, \ldots, k_{j}$. The first and last knot positions, $\bar{z}_{1}$ and $\bar{z}_{k_{j}}$, represent the minimum and maximum of all 
possible $z$ values, respectively. In vector notation, we can represent the smooth loadings by

$$
\Lambda_{j}=W_{j}^{\prime} \bar{\lambda}_{j}
$$

where $\Lambda_{j}$ is the $j$ th column of $\Lambda$ and $k_{j} \times N$ matrix of spline weights $W_{j}=\left(w_{1 j}, \ldots, w_{N j}\right)$ and with $w_{i j}$ defined as in (4). Instead of estimating the individual coefficients in the $j$ th column $\Lambda_{j}$, we estimate the smaller set of coefficients in $\bar{\lambda}_{j}$. For each column $\Lambda_{j}$, we can determine a different $z$ variable, a different number of knots $k_{j}$, a different set of knots $\bar{z}_{1}, \ldots, \bar{x}_{k}$ and a different coefficient vector $\bar{\lambda}_{j}$.

When a small number of knots $k_{j}$ is chosen, the factor loadings in the $j$ th column of $\Lambda$ exhibit a highly smooth pattern. In our approach it is not necessary that all columns $\Lambda$ are smooth. When the number of knots is equal to $N$, we have $k_{j}=N$ and $w_{i j}$ reduces to the $i$ th column of the identity matrix $I_{N}$ such that $N \times 1$ vector $\bar{\lambda}_{j}$ contains all coefficients for the $j$ th column of $\Lambda$. As a result, no smoothness restrictions are imposed on the factor loadings in $\Lambda_{j}$.

\subsection{Inclusion of macroeconomic variables}

The smooth dynamic factor model (SDFM) can be extended with a, possibly large, panel of macroeconomic time series variables. We denote the $k \times 1$ vector of macroeconomic variables at time $t$ by $x_{t}$. The SDFM model with the inclusion of the macro panel is given by

$$
\left(\begin{array}{l}
x_{t} \\
y_{t}
\end{array}\right)=\left(\begin{array}{l}
\mu_{x} \\
\mu_{y}
\end{array}\right)+\left[\begin{array}{cc}
\Gamma_{x x} & 0 \\
\Gamma_{y x} & \Lambda
\end{array}\right]\left(\begin{array}{c}
f_{t}^{x} \\
f_{t}
\end{array}\right)+\varepsilon_{t}, \quad \varepsilon_{t} \sim \operatorname{NID}(0, H), \quad t=1, \ldots, n,
$$

where $\mu_{x}$ is a $k \times 1$ vector of constants, $\Gamma_{x x}$ is the $k \times r_{x}$ factor loading matrix, $\Gamma_{y x}$ is the $N \times r_{x}$ factor loading matrix, $f_{t}^{x}$ is an $r_{x}$-dimensional stochastic process, $\varepsilon_{t}$ is the $(k+N) \times 1$ disturbance vector and $H$ is an $(k+N) \times(k+N)$ variance matrix. The vector $\mu_{y}$ and matrix $\Lambda$ are defined as for the SDFM observation equation (1) in section 2.2 while the factors $f_{t}$ are uniquely associated with the observed yields. The loading matrix $\Gamma_{x x}$ establishes the link between the macro series in $x_{t}$ and the macro factors $f_{t}^{x}$ in the same way as for the general dynamic factor model of section 2.1. The relation between the macroeconomic factors and the yields is modelled through the full matrix $\Gamma_{y x}$. The Gaussian disturbance vector series $\varepsilon_{t}$ is serially uncorrelated as NID refers to normally and independently distributed. We further assume that the variance matrix of the observation disturbances $H$ is diagonal. It implies that the covariance between the variables in $y_{t}$ depends solely on the latent factor $f_{t}$. The 
signal equation is given by

$$
\left(\begin{array}{c}
f_{t}^{x} \\
f_{t}
\end{array}\right)=\left[\begin{array}{c}
U_{x} \\
U
\end{array}\right] \alpha_{t},
$$

where the fixed $r_{x} \times p$ matrix $U_{x}$ relates the dynamic factor $f_{t}^{x}$ with the $p$-dimensional stochastic state vector $\alpha_{t}$ as specified in (3). The interaction between $y_{t}$ and $x_{t}$ in this dynamic factor model structure may allow the interpretation of $x_{t}$ as a covariate time series panel for improving the fit and forecast function for $y_{t}$.

\subsection{Parameter estimation and signal extraction}

The dynamic factor model (1) - (3) is a special case of the linear state space model. For given values of the system matrices, we can use the Kalman filter and related methods to evaluate minimum mean square linear estimates (MMSLE) of the state vector at time $t$ given the observation sets $\left\{y_{1}, \ldots, y_{t-1}\right\}$ (prediction), $\left\{y_{1}, \ldots, y_{t}\right\}$ (filtering) and $\left\{y_{1}, \ldots, y_{n}\right\}$ (smoothing). A detailed treatment of state space methods is given by Durbin and Koopman (2001). The Kalman filter can also be used to evaluate the loglikelihood function via the prediction error decomposition. The maximum likelihood estimates of the unknown parameters are obtained via the numerical optimization of the loglikelihood function. To generate the results in this paper we used the BFGS algorithm to perform the optimization; see, for example, Nocedal and Wright (1999). An alternative approach would be to use the EM algorithm as developed for state space models by Watson and Engle (1983).

Computationally efficient versions of the Kalman filter have been developed for multivariate models; see, for example, Koopman and Durbin (2000). Furthermore, we can achieve considerable computational savings using the methods of Jungbacker and Koopman (2008). Their method first maps the set of observations $y_{t}$ into a set of vectors which have the same dimensions as the latent factors $f_{t}$ in $(2)$. We can then apply the Kalman filter to a typically lower dimensional "observation" vector. We have implemented this approach in our analysis. These efficient Kalman filter methods are also used to evaluate the closed form expressions for the score function given in Koopman and Shephard (1992). Despite of the large number of parameters involved, this combination of efficient Kalman filter methods and analytical score computations allows us to estimate parameters in a matter of seconds.

The general estimation methods can also be used for the SDFM. The number of knots and the knot positions are determined using a sequence of estimation and testing steps. Whether a knot coefficient should remain in the model can be determined by a Wald test or a likelihood ratio test procedures; see Jungbacker, Koopman, and van der Wel (2010) for the details of our parameter estimation procedure for the SDFM. 


\section{Empirical results}

We have constructed a monthly time series panel of unsmoothed Fama-Bliss zero yields for U.S. treasuries of different maturities between 1970 and 2009. We combine this panel with a data set of macroeconomic time series for the same sample period. The details of the data set are provided in section 3.1. The estimation results for the smooth dynamic factor model for the yield curve, as discussed in section 2.2, are presented in section 3.2. Section 3.3 presents the estimation results for the dynamic factor model of section 2.1 on the macro data set. In section 3.4, we discuss the estimation results for our macroeconomic smooth dynamic factor model of section 2.3. Finally, in section 3.5 we present the results of our forecasting study.

\subsection{Data description}

Our empirical study is based on a new data set of U.S. interest rates that is constructed in similar way as the data used in Diebold and Li (2006), and is also used by Jungbacker, Koopman, and van der Wel (2010). A panel of monthly time series of zero yields from the CRSP unsmoothed Fama and Bliss (1987) forward rates is constructed. We refer to Diebold and Li (2006) for a detailed discussion of the method that is used for the creation of this data set. The resulting balanced panel data set consists of 17 maturities over the period from January 1970 up to December 2009, we have $N=17$ and $n=480$. The maturities we analyze are 3, 6, 9, 12, 15, 18, 21, 24, 30, 36, 48, 60, 72, 84, 96, 108 and 120 months. Similar but shorter datasets have been considered by Diebold, Rudebusch, and Aruoba (2006), Christensen, Diebold, and Rudebusch (2010) and Bowsher and Meeks (2008).

In Panel A of Figure 1 we present a three-dimensional plot of the data set. The data plot suggests the presence of an underlying factor structure. Although the yield series vary heavily over time for each of the maturities, a strong common pattern in the 17 series over time is apparent. For most months, the yield curve is an upward sloping function of time to maturity. The overall level of the yield curve is mostly downward trending over time in our sample period. These findings are supported by the time series plots in Panel B of Figure 1. We also observe that volatility tends to be lower for the yields of bonds with a longer time to maturity.

Table 1 provides summary statistics for our dataset. For each of the 17 time series we report mean, standard deviation, minimum, maximum and a selection of autocorrelation and partial autocorrelation coefficients. The summary statistics confirm that the yield curve tends to be upward sloping and that volatility is lower for rates on the long end of the yield curve. In addition, there is a very high persistence in the yields: the first order autocorre- 
Figure 1: Yield Curves from January 1970 through December 2009

In this figure we show the U.S. Treasury yields over the period 1970-2009. We examine monthly data, constructed using the unsmoothed Fama-Bliss method. The maturities we show are $3,6,9,12,15,18,21,24,30,36,48,60,72,84,96,108$ and 120 months. Panel A presents a 3 -dimensional plot, Panel B provides time-series plots for selected maturities.

\section{(A) 3-Dimensional Term Structure Plot}

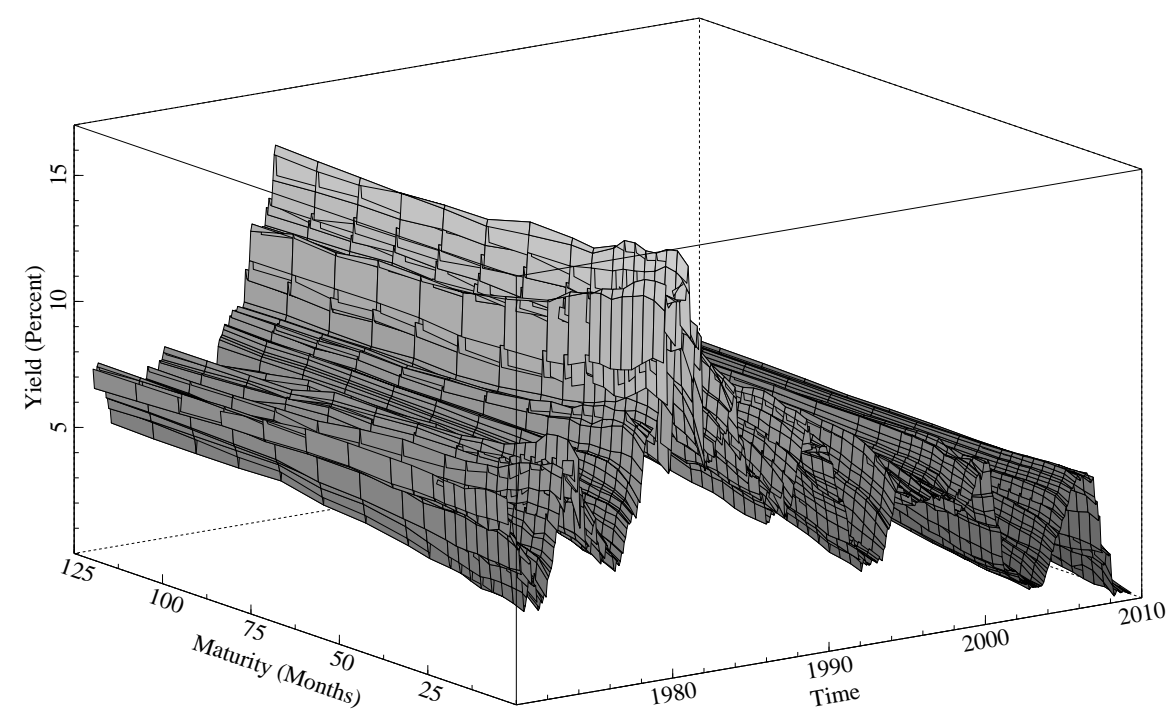

(B) Time-Series for Selected Maturities
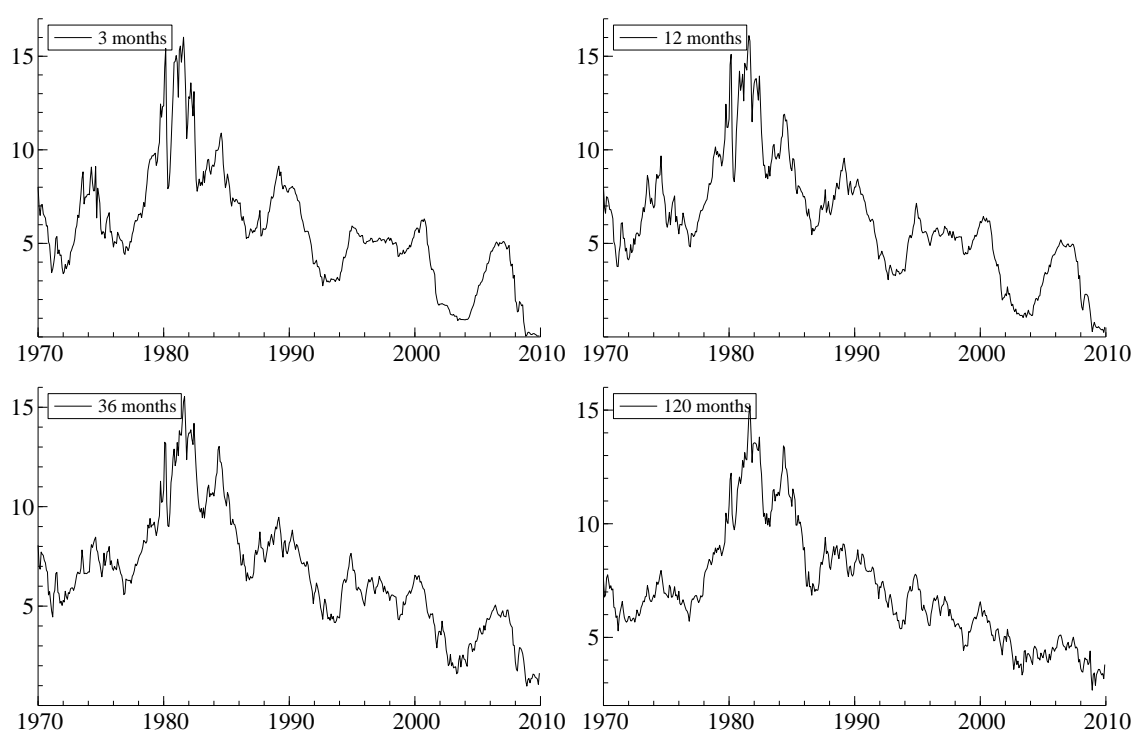
Table 1: Summary Statistics of Yield Curve Data

The table reports summary statistics for U.S. Treasury yields over the period 1970-2009. We examine monthly data, constructed using the unsmoothed Fama-Bliss method. Maturity is measured in months. In Panel A we show for each maturity mean, standard deviation $(S d)$, minimum, maximum and two (1 month and 12 month) autocorrelation (Acf, $\hat{\rho}(1)$ and $\hat{\rho}(12)$ respectively) and two (2 month and 12 month) partial-autocorrelation $($ Pacf, $\hat{\alpha}(2)$ and $\hat{\alpha}(12))$ coefficients. In Panel B we show the correlation matrix for some selected maturities.

\begin{tabular}{|c|c|c|c|c|c|c|c|c|}
\hline \multicolumn{9}{|c|}{ Panel A: Summary Statistics } \\
\hline \multirow[b]{2}{*}{ Maturity } & \multirow[b]{2}{*}{ Mean } & \multirow[b]{2}{*}{$\mathrm{Sd}$} & \multirow[b]{2}{*}{ Min } & \multirow[b]{2}{*}{ Max } & \multicolumn{2}{|c|}{ Acf } & \multicolumn{2}{|c|}{ Pacf } \\
\hline & & & & & $\hat{\rho}(1)$ & $\hat{\rho}(12)$ & $\hat{\alpha}(2)$ & $\hat{\alpha}(12)$ \\
\hline 3 & 5.77 & 3.07 & 0.04 & 16.02 & 0.98 & 0.75 & -0.11 & -0.06 \\
\hline 6 & 5.97 & 3.09 & 0.15 & 16.48 & 0.98 & 0.76 & -0.13 & -0.11 \\
\hline 9 & 6.08 & 3.09 & 0.19 & 16.39 & 0.98 & 0.77 & -0.14 & -0.12 \\
\hline 12 & 6.17 & 3.05 & 0.25 & 16.10 & 0.98 & 0.78 & -0.15 & -0.13 \\
\hline 15 & 6.25 & 3.03 & 0.38 & 16.06 & 0.98 & 0.78 & -0.15 & -0.13 \\
\hline 18 & 6.32 & 3.01 & 0.44 & 16.22 & 0.98 & 0.79 & -0.15 & -0.14 \\
\hline 21 & 6.39 & 2.99 & 0.53 & 16.17 & 0.98 & 0.80 & -0.14 & -0.15 \\
\hline 24 & 6.42 & 2.94 & 0.53 & 15.81 & 0.98 & 0.80 & -0.16 & -0.14 \\
\hline 30 & 6.51 & 2.88 & 0.82 & 15.43 & 0.98 & 0.81 & -0.13 & -0.13 \\
\hline 36 & 6.60 & 2.83 & 0.98 & 15.54 & 0.98 & 0.81 & -0.13 & -0.11 \\
\hline 48 & 6.76 & 2.75 & 1.02 & 15.60 & 0.98 & 0.82 & -0.11 & -0.12 \\
\hline 60 & 6.85 & 2.67 & 1.56 & 15.13 & 0.99 & 0.83 & -0.10 & -0.12 \\
\hline 72 & 6.96 & 2.64 & 1.52 & 15.11 & 0.99 & 0.84 & -0.11 & -0.12 \\
\hline 84 & 7.03 & 2.57 & 2.18 & 15.02 & 0.99 & 0.84 & -0.12 & -0.11 \\
\hline 96 & 7.07 & 2.53 & 2.11 & 15.05 & 0.99 & 0.85 & -0.12 & -0.12 \\
\hline 108 & 7.10 & 2.52 & 2.15 & 15.11 & 0.99 & 0.85 & -0.14 & -0.14 \\
\hline 120 & 7.07 & 2.46 & 2.68 & 15.19 & 0.99 & 0.84 & -0.12 & -0.13 \\
\hline
\end{tabular}

Panel B: Correlation Matrix

for Selected Maturities

\begin{tabular}{lrrrrr}
\hline Maturity & 3 & 12 & 36 & 60 & 120 \\
\hline 3 & 1.00 & 0.99 & 0.96 & 0.93 & 0.90 \\
12 & & 1.00 & 0.98 & 0.96 & 0.93 \\
36 & & & 1.00 & 1.00 & 0.98 \\
60 & & & & 1.00 & 0.99 \\
120 & & & & & 1.00 \\
\hline
\end{tabular}


lation for all maturities is above 0.97 for each maturity. Even the twelfth autocorrelation coefficient can be as high as 0.85 . The partial autocorrelation function suggests that autoregressive processes of limited lag order will fit the data well since only the first coefficient is significant for most maturities while the second lag coefficients are relatively small. (to preserve space we display a selection of coefficients). In Panel B of the Table 1 we present the sample correlations between yields of a selected number of maturities. The correlations are mostly above 0.9 , in accordance with the strong common patterns in the movements of the different yields that we observe in Figure 1.

\section{Table 2: Overview of Macroeconomic Series}

The table lists the sectors in our data set on macroeconomic variables, and the number of available series in each sector. The data is an updated version of the Stock and Watson (2005) data. We focus on series that are available over our entire sample period of 1970-2009, and list both the original number of series in Stock and Watson (2005) (column label SW2005) and our study (Update).

\begin{tabular}{llrr}
\hline \multicolumn{3}{c}{ Overview of macro series } \\
\hline & & \multicolumn{2}{c}{$\#$ Series } \\
\cline { 2 - 4 } Description & SW2005 & Update \\
\hline A & Real Output and Income & 18 & 18 \\
B & Employment and Hours & 30 & 30 \\
C & Real Retail, Manufacturing and Trade Sales & 2 & 2 \\
D & Consumption & 1 & 1 \\
E & Housing Starts and Sales & 10 & 10 \\
F & Real Inventories and Inventory-Sales Rations & 3 & 3 \\
G & Orders and Unfilled Orders & 6 & 6 \\
H & Stock Prices & 4 & 2 \\
I & Exchange Rates & 5 & 5 \\
J & Interest Rates and Spreads & 17 & 0 \\
K & Money and Credit Quantity Aggregates & 11 & 9 \\
L & Price Indexes & 21 & 20 \\
M & Average Hourly Earnings & 3 & 3 \\
N & Miscellanea & 1 & 1 \\
\hline & TOTAL & 132 & 110 \\
\hline
\end{tabular}

We complement the yield curve data with a panel of macroeconomic time series. This macroeconomic panel is originally considered by Stock and Watson (2002). It was then modified and the time span has been extended by Stock and Watson (2005) and Exterkate, Van Dijk, Heij, and Groenen (2010), we performed a further extension of the sample period for our empirical study. The data consists of 132 variables that we have grouped in 14 
different type of variables, following the Stock and Watson (2002) classification that includes Real Output, Employment, Consumption, Stock Prices, etc. A complete overview of the 14 different groups of variables and the number of variables in each group are presented in Table 2. Our balanced macroeconomic panel consists of a high variety of variables that should provide an accurate indication of the U.S. economic climate over the period from January 1970 up to December 2009. We have taken out all interest rate related variables because we want to investigate how macroeconomic information can help to improve yield curve forecasting. For this reason and due to the unavailability of 5 other series, we have in total 110 series in our macroeconomic panel which gives $k=110$ and $n=480$. We transform all macroeconomic variables following Stock and Watson (2005) to obtain stationary series, and standardize these series by subtracting the mean and dividing by the sample standard deviation of each series.

\subsection{Estimation of smooth dynamic factors without macro series}

The models considered in this study belong to the class of dynamic factor models (1)-(3) and include a total of three latent yield factors, that is $r=3$. Here we follow a growing number of studies that find three factors adequate for explaining most of the variation in the cross-section of yields; see, for example, Litterman and Scheinkman (1991), Bliss (1997) and Diebold and Li (2006). Other studies have recommended more factors; see the discussion in De Pooter (2007).

Our time series panel of U.S. interest rates for 17 maturities is represented by $y_{t}$ and is modelled as in (1) with a $17 \times 1$ vector of constants $\mu_{y}$, a full $17 \times 3$ loading matrix $\Lambda$ as specified in (5), a $17 \times 17$ diagonal variance matrix $H$. For the identification of all parameters in the DFM and to keep the $\operatorname{VAR}(1)$ coefficient matrices unrestricted, we restrict the three rows corresponding to maturities of 1 (first row), 30 (ninth row) and 120 months (last row) in $\Lambda$. In particular, this set of three rows is set equal to

$$
\lambda_{1, \cdot}=(1,1,0), \quad \lambda_{9, \cdot}=\left(1, \frac{1}{2}, 1\right), \quad \lambda_{17, \cdot}=(1,0,0) .
$$

These restrictions facilitate the interpretation of the factors as level, slope and curvature, in a similar way, to some extent, as those from the Nelson and Siegel (1987) yield curve. We have noticed that the estimation results are not qualitatively different when we consider other sets of restrictions (for example, the rows of the identity matrix $I_{3}$ ) since we can rotate the factors such that another set of restrictions is implied. The dynamic specification for the three factors in $f_{t}$ are modelled jointly by a vector autoregressive process of lag order 1 , the 
$\operatorname{VAR}(1)$ model, and is given by

$$
f_{t+1}=\Phi f_{t}+\eta_{t}, \quad \eta_{t} \sim \operatorname{NID}(0, Q), \quad t=1, \ldots, n
$$

which can be expressed as in (3) with $\mu_{\alpha}=0, T=\Phi, U=R=I_{r}$ and $\alpha_{t}=f_{t}$. We denote this model by $\operatorname{VAR}(1)$. In our empirical study, we consider the $\operatorname{VAR}(1)$ specification for the factor process in all model specifications. This choice is the same as in related studies such as Diebold, Rudebusch, and Aruoba (2006); the exception is Bowsher and Meeks (2008) where a cointegrated VAR system with multiple lags is considered for the factors.

For the smooth dynamic factor model we take the optimal model that is obtained in Jungbacker, Koopman, and van der Wel (2010); we estimate the parameters for this model by the method of maximum likelihood. Based on the likelihood ratio test procedure, our established model consists of a total of 20 knots in the $\Lambda$ matrix. The estimated loadings associated with the first factor are very close to unity and therefore we can interpret the first factor as the level. The loading estimates for the second factor are smoothly descending from one to zero for interest rates of ascending maturity. This is the typical Nelson and Siegel (1987) shape for their second factor which they associate with the slope of the yield curve. The third Nelson-Siegel factor is designed as the curvature of the yield curve with the associated loading pattern given by an asymmetric, reverse U-shape. Our loading estimates for the third factor also admit to this pattern and therefore we can interpret the third factor in $f_{t}$ as the curvature of the yield curve. In case of the DFM, the loading restrictions in (7) have facilitated the level-slope-curvature (LSC) interpretation of the three factors. When other loading restrictions were considered, the estimation results are not different since appropriate factor rotations can be carried out to obtain the same loadings. Jungbacker, Koopman, and van der Wel (2010) show favorable performance of the smooth dynamic factor model over Nelson and Siegel (1987) and Bowsher and Meeks (2008) models. This latter model can be seen as a restricted version of the smooth dynamic factor model in which the number of knots is equal to the number of factors and the knot coefficients become the time-varying factors; see Harvey and Koopman (1993) for a detailed description and an electricity load forecasting application.

The autoregressive coefficient matrix $\Phi$ and the variance matrix $Q$ in (8) are estimated jointly with $\mu_{y}, \Lambda$ and $H$ in (1) by the method of maximum likelihood. The estimates for $\Phi$ are presented in Panel A of Table 3. The leading diagonal of the estimated $\Phi$ contains values close to unity while the off-diagonal elements are all smaller than 0.1 in absolute value. It indicates that the factors are highly persistent over time.

Next we analyze the factor loadings of the SDFM model together with the estimated 
Table 3: Estimated Transition Matrices

In this table we present the estimated transition matrices for the dynamic factor models for the yield curve, macroeconomic series, and the dynamic factor model with both yield and macro factors. In Panel A we report results for the SDFM for the yield curve, in Panel B for the DFM for the macroeconomic series, and Panel C for the macroeconomic SDFM.

Panel A: Transition Matrix

of SDFM for Yield Curve

\begin{tabular}{rrrr}
\hline & $f_{1, t-1}$ & $f_{2, t-1}$ & $f_{3, t-1}$ \\
\hline$f_{1, t}$ & 1.005 & 0.017 & -0.062 \\
$f_{2, t}$ & -0.004 & 0.968 & 0.206 \\
$f_{3, t}$ & 0.013 & -0.007 & 0.819 \\
\hline
\end{tabular}

Panel B: Transition Matrix

of DFM for Macro Series

\begin{tabular}{rrrr}
\hline & $f_{1, t-1}$ & $f_{2, t-1}$ & $f_{3, t-1}$ \\
\hline$f_{1, t}$ & 0.157 & 0.363 & -0.098 \\
$f_{2, t}$ & 0.209 & 0.026 & -0.019 \\
$f_{3, t}$ & -0.167 & -0.247 & 0.954 \\
\hline
\end{tabular}

Panel C: Transition Matrix of Macroeconomic SDFM

\begin{tabular}{rrrrrrr}
\hline & $f_{1, t-1}$ & $f_{2, t-1}$ & $f_{3, t-1}$ & $f_{4, t-1}$ & $f_{5, t-1}$ & $f_{6, t-1}$ \\
\hline$f_{1, t}$ & 0.997 & 0.026 & -0.127 & -0.004 & 0.118 & 0.003 \\
$f_{2, t}$ & -0.004 & 0.962 & 0.073 & 0.055 & 0.049 & -0.003 \\
$f_{3, t}$ & 0.008 & -0.003 & 0.883 & 0.009 & -0.022 & 0.000 \\
$f_{4, t}$ & 0.022 & -0.133 & 0.094 & 0.119 & 0.344 & -0.103 \\
$f_{5, t}$ & -0.019 & -0.071 & 0.263 & 0.195 & 0.016 & -0.026 \\
$f_{6, t}$ & 0.021 & 0.176 & -0.442 & -0.085 & -0.216 & 0.976 \\
\hline
\end{tabular}


Figure 2: Smooth Dynamic Factor Model for Yield Curve

In this figure we present the latent factors obtained from the Smooth Dynamic Factor Model (SDFM) for the yield curve. Panel A presents the time series from the 3 obtained latent factors. Panel B shows the correlation between each of these latent factors and the maturity.

(A) Latent Factors in SDFM for Yield Curve
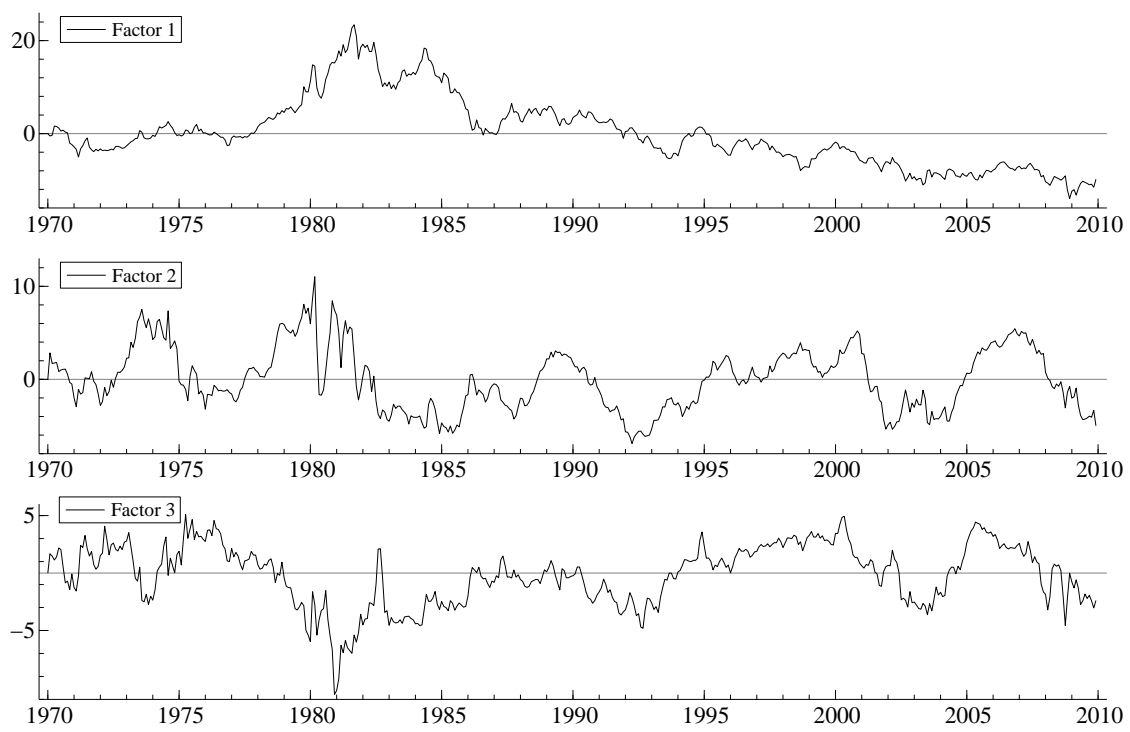

(B) Correlation between Latent Factors and Yield per Maturity
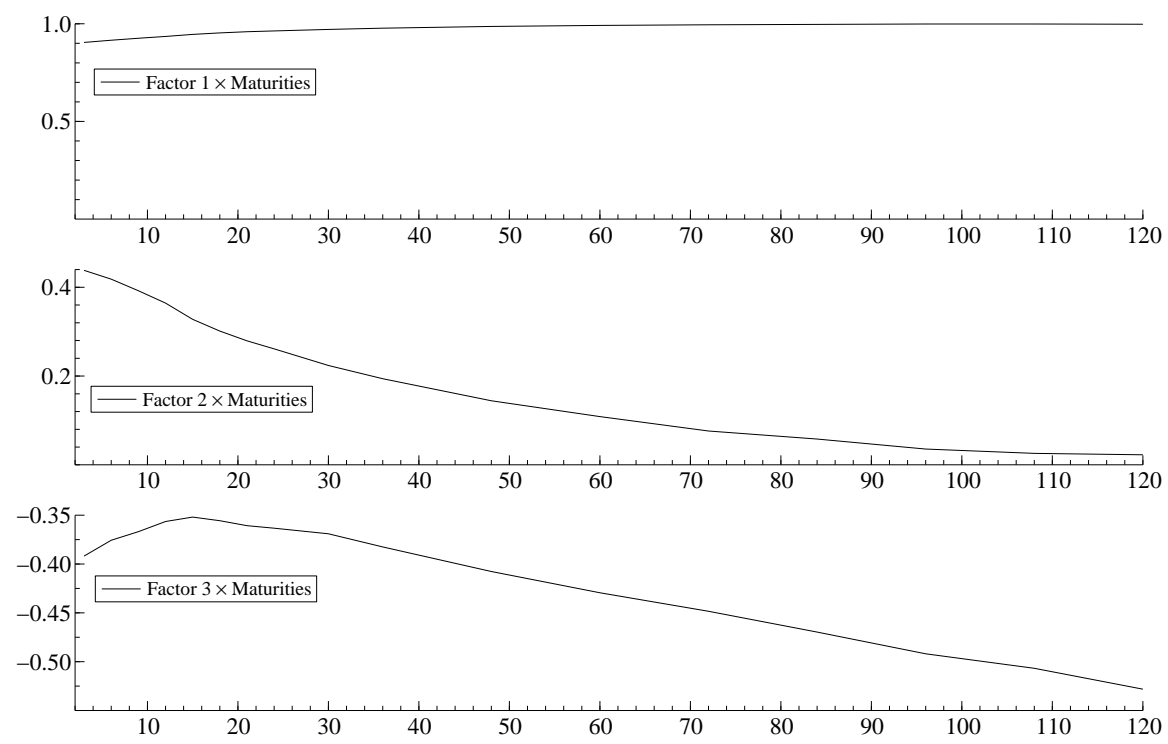
factors. The imposed restrictions lead to a factor loading structure that is very similar to the Nelson and Siegel (1987) loading structure with the level, slope and curvature interpretations of the factors. The three factor estimates are presented in Panel A of Figure 2. The first graph is for the level and it shows the general evolution of the interest rates through the time period 1970 - 2009. The estimated slope factor in the second graph of Panel A is a relatively smooth time process with characteristics that can be associated with the business cycle. The curvature factor is more noisy process although it clearly picks up some dynamic features in the data.

To emphasize the smooth loading structure between the data and the factors, we present correlation statistics with the interest rates of the 17 different maturities and the three factors in Panel B of Figure 2. It confirms the interpretation of the factors and the smooth nature of the (cross-sectional) factor loadings. The level factor is almost equally correlated with the interest rates for all maturities while the slope factor is increasingly correlated with the interest rates as the associating maturity is increasing. The third factor has loadings that typically represent the curvature factor of the Nelson-Siegel yield curve.

\subsection{A dynamic factor analysis for the macro series}

In Table 2 we have grouped the 110 macroeconomic variables of our time series panel into 14 groups. To provide some insight in the information content of the panel, we have carried out a dynamic factor analysis for these series. We consider three factors for the 110 variables and we have estimated the parameters in the model by the method of maximum likelihood. The resulting estimated factors from the analysis are obtained from the Kalman filter and smoother. We have presented the three factor estimates in Panel A of Figure 3. The first and second factors are somewhat noisy indicators while the third factor is much more persistent and appears to show some business cycle features. For example, the third factor has some pronounced positive deviations from zero which are clearly associated with recession years in the U.S. Most pronounced is the positive deviations from 2007 onwards which may represent the recent financial crisis.

It is of interest to investigate which macroeconomic variables are associated with the estimated factors. For this purpose, we present the correlations of the estimated factors with the macroeconomic variables in Panel B of Figure 3. From these graphs we can learn that the first factor is related to many economic variables but most pronouncedly with the first groups that particularly represent the real economy. The second factor is also correlated with the real economy variables but most prominently with price indices. The third factor (or the recession indicator) is mostly correlated with the variables working hours and housing 
Figure 3: Dynamic Factor Model for Macroeconomic Series

In this figure we present the latent factors obtained from the Dynamic Factor Model (DFM) for the macroeconomic series. Panel A presents the time series from the 3 obtained latent factors. Panel B shows the correlation between each of these latent factors and the maturity.

(A) Latent Factors in DFM for Macroeconomic Series
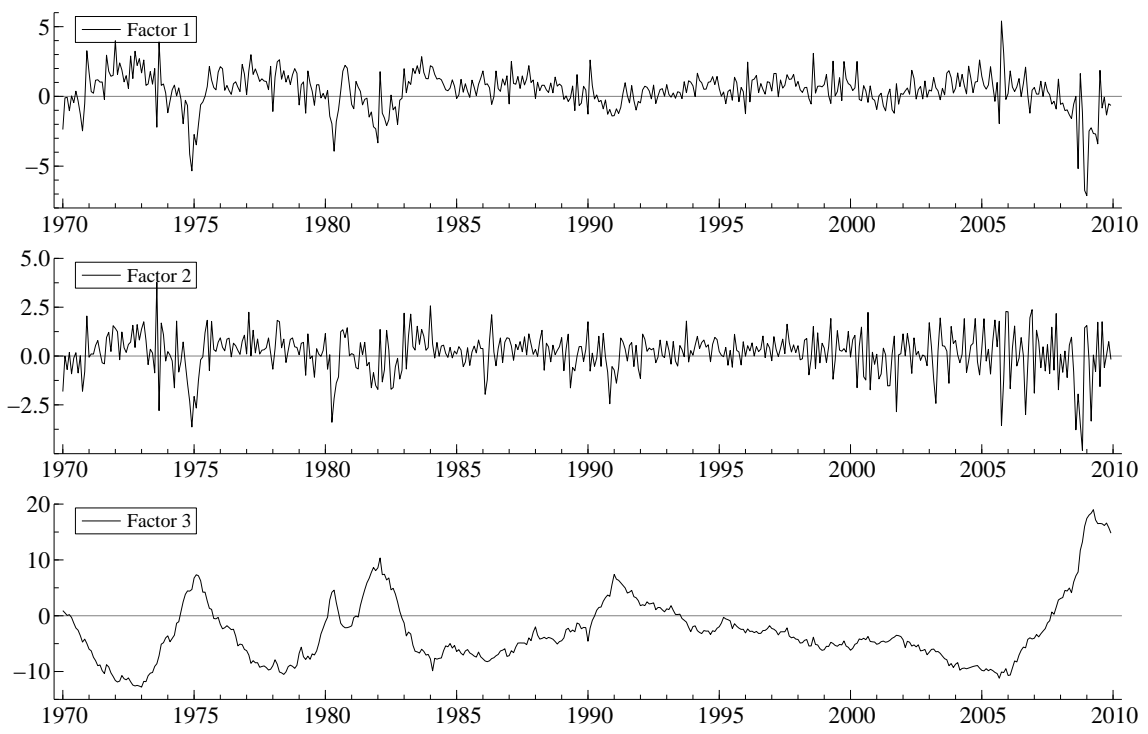

(B) Correlation between Latent Factors and Individual Macroeconomic Series
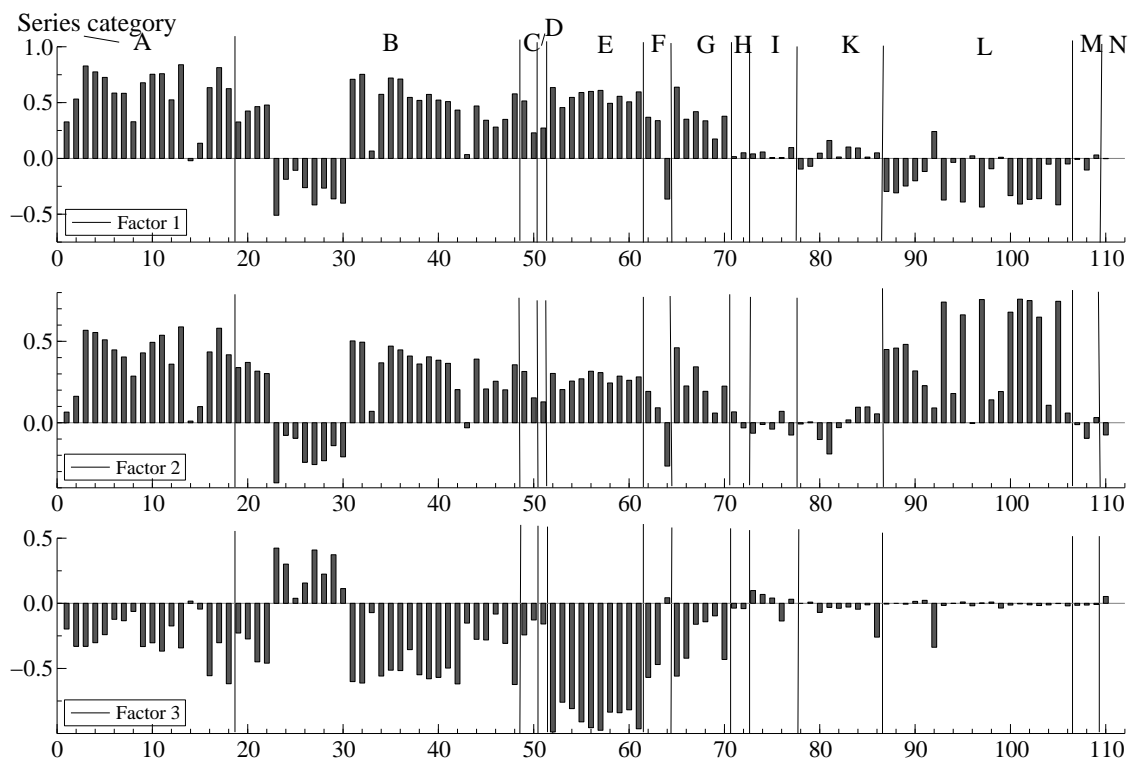
starts and sales. These variables are especially indicative of the recession years and the financial crisis.

The persistence of the first two factors is moderate, but high for the third factor. We report the estimates of the coefficients in the transition matrix $\Phi$ of the dynamic factor model in Panel B of Table 3. The estimates of the leading diagonal of $\Phi$ confirm the persistence levels of the three factors. The off-diagonal elements of $\Phi$ are all smaller than 0.25 in absolute values except the coefficient for the first factor that appears to rely on the lagged second factor. These relations may not lead to clear interpretations, they may however improve the forecast precision.

\subsection{A macroeconomic smooth dynamic factor analysis}

Next we present the estimation results for our macroeconomic smooth dynamic factor model as specified by (6) and discussed in section 2.3. The factor loading structures are similar to the presented results for our separate analyses. The loading matrix $\Lambda$ is subject to the Nelson-Siegel smooth cross-sectional structure while the factor loadings for $\Gamma_{x x}$ and $\Gamma_{y x}$ are not subject to restrictions (other than the identifying restrictions in $\Gamma_{x x}$ ) and all elements are estimated by the method of maximum likelihood together with the other unknown parameters. We remain to have three factors in $f_{t}$ for the yield curve (level, slope and curvature) and three macro factors in $f_{t}^{x}$. In total we have six factors which we model by a VAR(1) process. The estimated coefficients in the $6 \times 6$ matrix $\Phi$ are reported in panel $\mathrm{C}$ of Table 3. The estimates of the leading diagonal of $\Phi$ are all close to one for $f_{t}$ while those for $f_{t}^{x}$ have values between 0.02 and 0.98 . The estimated off-diagonal elements of $\Phi$ range between 0 and 0.44 in absolute values. Interestingly, the largest (in absolute sense) off-diagonal entry in $\Phi$ is the relation between the 3rd macroeconomic variable and the curvature factor. It highlights the interaction between the variables. It may also imply that the macroeconomic variables can be useful for the forecasting of the yield curve.

In panel A of Figure 4 we present the estimated factors. The first three factors represent the estimated $f_{t}$ and the next three factors are for $f_{t}^{x}$. The estimated factors appear overall to be more persistent than the individual estimates for $f_{t}$ and $f_{t}^{x}$. However, the same patterns are apparent. For example, we can recognize the recession periods in the second and sixth factor estimates. In particular, the sixth factor appears to be strongly affected by the recent financial crisis.

To obtain a more detailed insight in the associations of the factors with the yields and the macroeconomic variables, we compute the correlations for the estimated factors on one side and the yield and macro variables on the other side. The correlations of the factors 
Figure 4: Macroeconomic Smooth Dynamic Factor Model for Yield Curve In this figure we present the latent factors obtained from the macroeconomic Smooth Dynamic Factor Model for the yield data that includes the macroeconomic series. Panel A presents the time series from the 6 obtained latent factors (3 yield factors and 3 macro factors). Panels B and C show the correlation between each of these latent factors and the yield curve (Panel B) and macro series (Panel C).

(A) Latent Factors in Macroeconomic SDFM
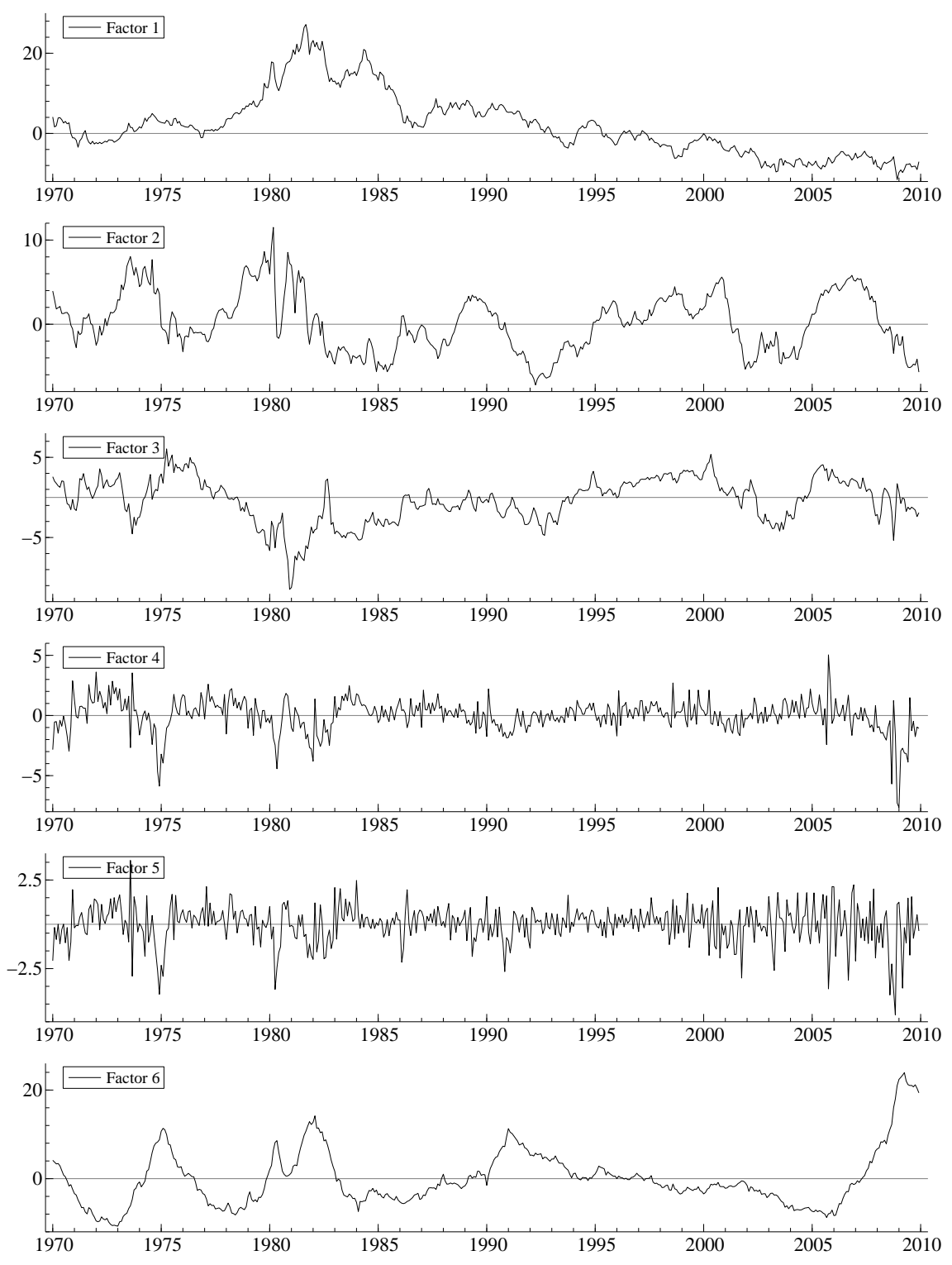
(B) Correlation between Latent Factors and Yield per Maturity
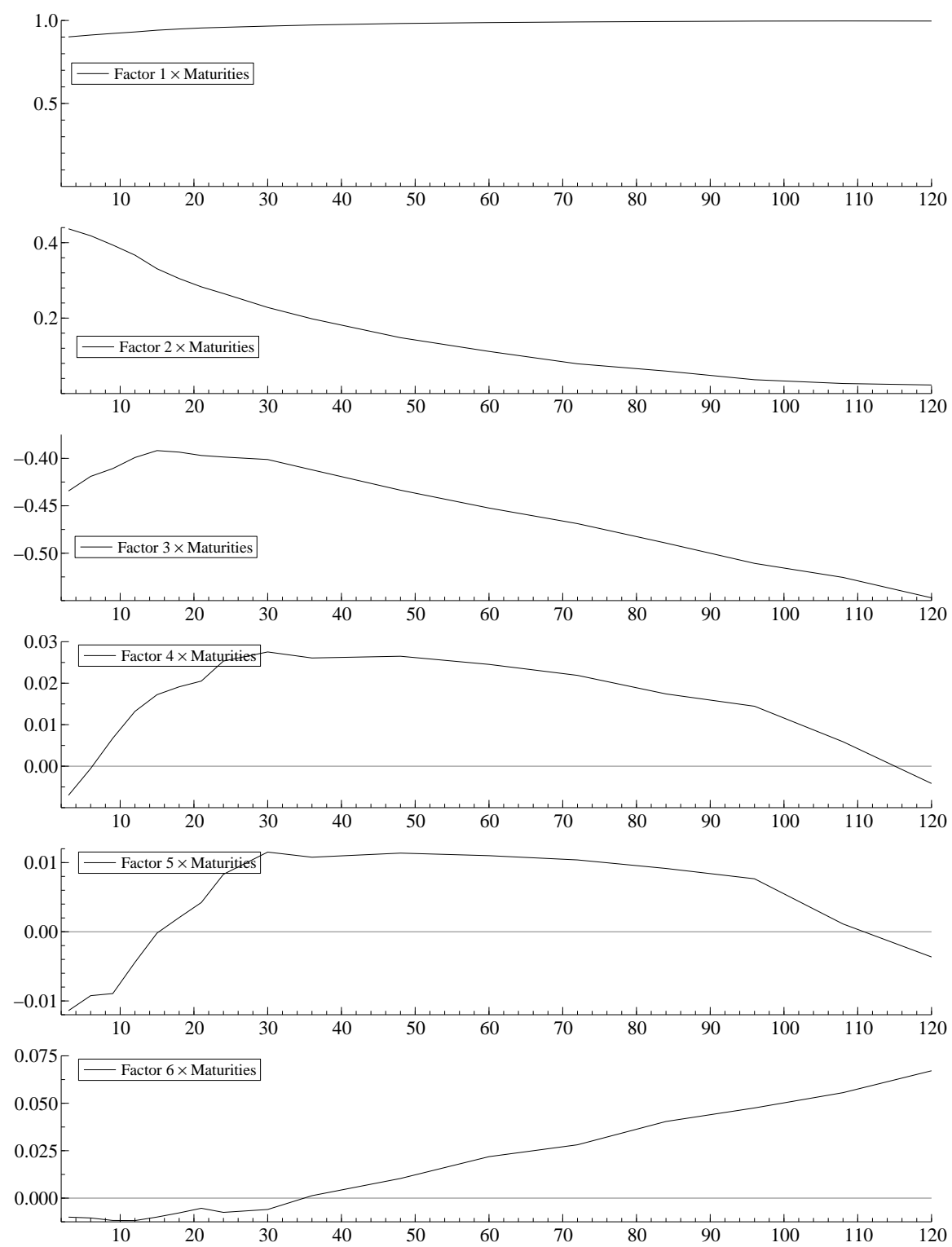
(C) Correlation between Latent Factors and Individual Macroeconomic Series
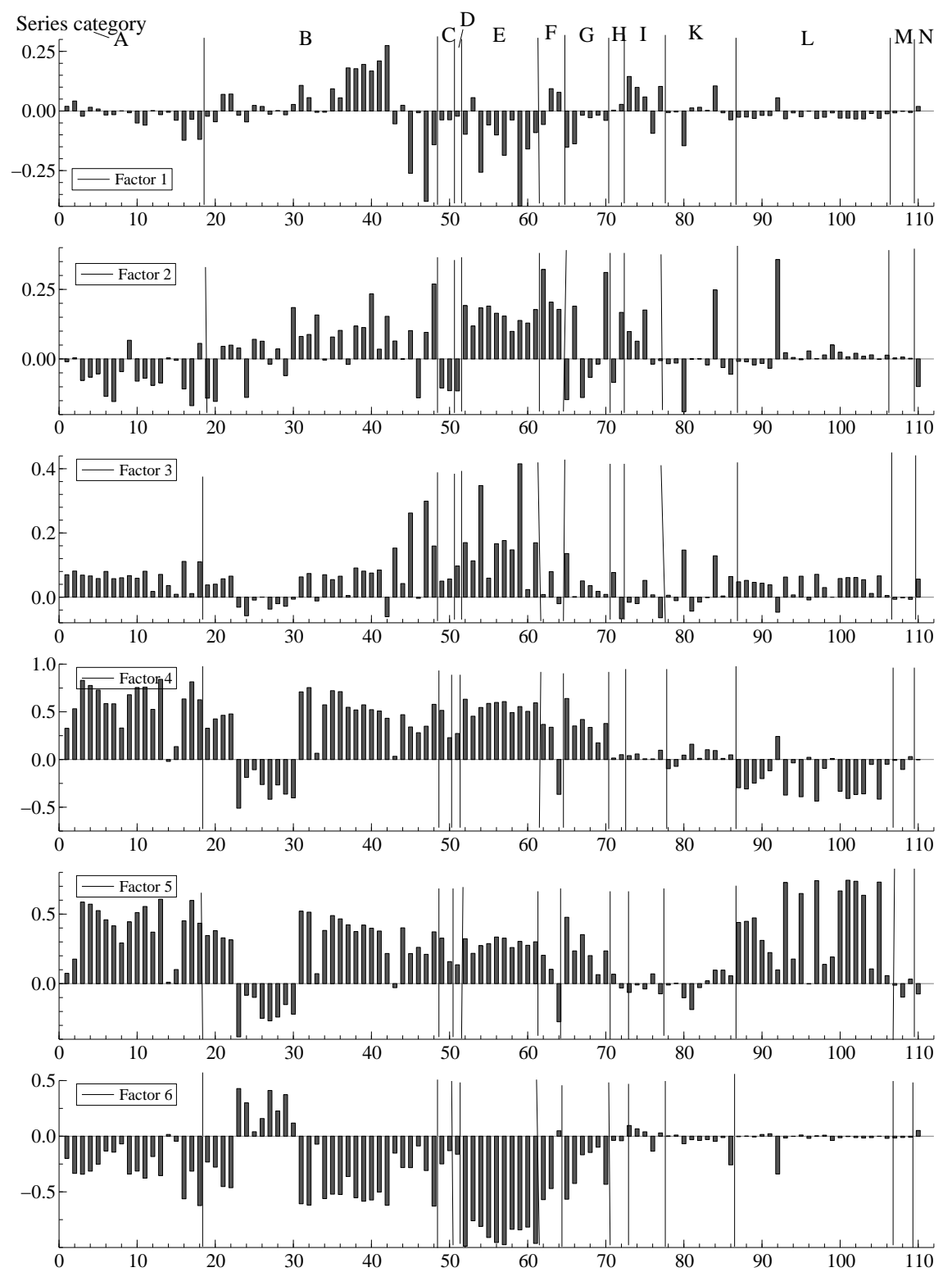
with the yield variables are presented in panel B of Figure 4. These graphs show that the factors in $f_{t}$ still have the level, slope and curvature interpretation for the yield curve. It is interesting to observe that the macro factors in $f_{t}^{x}$ are all associated with either the slope or curvature of the yield curve. We may interpret the slope of the yield as the spread in interest rates. The spread is often used as a predictor of economic recessions. This analysis confirms the strong association of the yield slope with macroeconomic variables. We can also conclude from panel B of Figure 4 that the interest rates associated with short maturities do not depend heavily on the macro factors. They appear to have a much stronger impact on medium maturity interest rates.

The correlations of the factors with the macroeconomic variables are presented in panel $\mathrm{C}$ of Figure 4. These correlations can provide insights into the impact of the factors on macroeconomic variables. The level yield factor is highly sensitive to employment and hours, and housing starts and sales. The slope yield factor is also related to these two categories but also to real inventories and inventory-sales rations and orders and unfilled orders. The curvature of the yield is mostly correlated with variables in the employment and hours, and housing starts and sales categories, of which the correlation with housing starts is over $40 \%$. The fourth factor represents the overall real economy. The money volume variables are particularly affected by the fifth factor while housing starts and sales are captured by the sixth factor. In essence all factors remain to keep their interpretation when we compare these results with the earlier results obtained from our earlier separate dynamic factor analyses. Next we will investigate the forecasting performances of the different dynamic factor analyses.

\subsection{Forecasting results}

To investigate the out-of-sample fit performance of our macroeconomic SDFM specification for our panel time series of U.S. interest rates, we carry out a forecasting exercise. We forecast the full yield curve (the interest rates for 17 times to maturity) up to 24 months ahead. We forecast the yield curve for the months from January 1994 up to December 2009. To obtain the 24 month ahead forecast of the yield curve for January 1994, we estimate the parameters in the four different models using the observations of the time series panel from January 1970 up to January 1992. The 23 month ahead forecast for January 1994 and the 24 month ahead forecast for February 1994 are obtained by estimating the parameters in the four different models based on the data from January 1970 up to February 1992. In this way we compute the forecasts, and the corresponding forecast errors, for $h=1,6,12,18,24$ months ahead. We record the forecast errors for each forecast horizon, for each maturity and for each model. Then we compute the root mean square forecast error (RMSFE) for all these cases. The 
highlights of our results for this extensive forecasting study are reported in Table 4 . The reestimation of the parameters in the different models is an extensive computational task but it remains feasible when using the methods discussed in section 2.4. For simplicity, we fix the number of knots for different sample periods and we also keep the knot positions as obtained from the knot selection procedure for the smooth dynamic factor models. The selection is based on the full sample. Alternative strategies where we, for example, yearly repeat the knot selection procedure have yielded similar forecasting results. In our analysis, we compare the macroeconomic smooth dynamic factor model to a SDFM without macroeconomic factors, a DFM for the yield curve without the smoothness restrictions, the Nelson and Siegel (1987) model casted in state space form, and the functional signal plus noise model of Bowsher and Meeks (2008).

In Panel A of Table 4 we report the forecasting results for the expanding window by means of the RMSFEs for the times to maturity 3, 12, 36, 60, 120 months. The forecasts produced by the macroeconomic SDFM, for the 3-, 12- and 36-month interest rate provide the lowest RMFSE amongst those obtained by other models considered. For the 5-year interest rate, and only for the forecasting horizon of 24 months, the unrestricted DFM performs best. For the 10-year interest rate, the RMSFE for the DFM is sometimes best and at a few other occasions it is best for the Nelson and Siegel (1987) model. The inclusion of macroeconomic factors in the smooth dynamic factor model appears to bring us lower RMSFEs for most interest rates and for most forecast horizons. In Panel B we examine the robustness of the forecasting improvement. We show the RMSFEs for the periods 1994-1998, 1999-2003 and 2004-2009. In the first two subsamples the lowest RMSFEs are produced mostly by the SDFM and macroeconomic SDFM, while sometimes it is the Nelson and Siegel (1987) or DFM that has the best performance. In the last subsample the macroeconomic SDFM performs remarkably well. We can conclude overall that the incorporation of macroeconomic variables in the SDFM improves the performance for forecasting the U.S. term structure of interest rates. 
Table 4: Forecasting Performance of Factor Models without macro series

In these tables we present the forecasting performance of the various models. We report the root mean square forecast error (RMSFE) for the macroeconomic SDFM, SDFM without macroeconomic variables, general DFM without smoothness restrictions, Nelson-Siegel in SSF (NS-SSF) and Functional Signal plus Noise (FSN) models. In Panel A we show results for the 1994-2009 sample, in Panel B for three different subperiods (1994-1998, 1999-2003 and 2004-2009). For each model the RMSFE is reported for 3 month, 1, 3,5 and 10 year maturities, and $h=1,6,12,18$ and 24 -month ahead forecasts.

\begin{tabular}{|c|c|c|c|c|c|}
\hline \multicolumn{6}{|c|}{ Panel A: Forecasting Performance 1994-2009 } \\
\hline & 3 month & 1 year & 3 year & 5 year & 10 year \\
\hline \multicolumn{6}{|c|}{ Macroeconomic SDFM } \\
\hline$h=1$ & 0.272 & 0.263 & 0.311 & 0.308 & 0.302 \\
\hline$h=6$ & 0.885 & 0.958 & 0.920 & 0.856 & 0.689 \\
\hline$h=12$ & 1.430 & 1.490 & 1.361 & 1.225 & 0.966 \\
\hline$h=18$ & 1.913 & 1.932 & 1.709 & 1.504 & 1.128 \\
\hline$h=24$ & 2.378 & 2.343 & 2.023 & 1.768 & 1.327 \\
\hline \multicolumn{6}{|c|}{ SDFM } \\
\hline$h=1$ & 0.316 & 0.275 & 0.322 & 0.316 & 0.302 \\
\hline$h=6$ & 0.969 & 1.021 & 0.971 & 0.881 & 0.681 \\
\hline$h=12$ & 1.639 & 1.652 & 1.454 & 1.272 & 0.957 \\
\hline$h=18$ & 2.127 & 2.105 & 1.804 & 1.557 & 1.143 \\
\hline$h=24$ & 2.477 & 2.444 & 2.087 & 1.803 & 1.339 \\
\hline \multicolumn{6}{|l|}{ DFM } \\
\hline$h=1$ & 0.328 & 0.273 & 0.320 & 0.315 & 0.300 \\
\hline$h=6$ & 0.959 & 1.008 & 0.958 & 0.871 & 0.679 \\
\hline$h=12$ & 1.614 & 1.626 & 1.426 & 1.246 & 0.943 \\
\hline$h=18$ & 2.094 & 2.067 & 1.759 & 1.512 & 1.110 \\
\hline$h=24$ & 2.435 & 2.397 & 2.028 & 1.741 & 1.288 \\
\hline \multicolumn{6}{|c|}{ NS-SSF } \\
\hline$h=1$ & 0.347 & 0.271 & 0.321 & 0.319 & 0.293 \\
\hline$h=6$ & 0.998 & 1.004 & 0.965 & 0.878 & 0.696 \\
\hline$h=12$ & 1.671 & 1.622 & 1.440 & 1.259 & 0.981 \\
\hline$h=18$ & 2.166 & 2.064 & 1.781 & 1.532 & 1.169 \\
\hline$h=24$ & 2.526 & 2.400 & 2.061 & 1.772 & 1.367 \\
\hline \multicolumn{6}{|l|}{ FSN } \\
\hline$h=1$ & 0.379 & 0.265 & 0.316 & 0.310 & 0.295 \\
\hline$h=6$ & 0.969 & 0.989 & 0.953 & 0.886 & 0.721 \\
\hline$h=12$ & 1.665 & 1.614 & 1.437 & 1.281 & 1.010 \\
\hline$h=18$ & 2.183 & 2.073 & 1.803 & 1.581 & 1.205 \\
\hline$h=24$ & 2.553 & 2.414 & 2.091 & 1.827 & 1.393 \\
\hline
\end{tabular}


Panel B: Forecasting Performance for Subperiods

\begin{tabular}{|c|c|c|c|c|c|c|c|c|c|c|c|c|c|c|c|}
\hline \multicolumn{16}{|c|}{ Panel B: Forecasting Performance for Subperiods } \\
\hline & \multicolumn{5}{|c|}{ Forecasting Performance 1994-1998 } & \multicolumn{5}{|c|}{ Forecasting Performance 1999-2003 } & \multicolumn{5}{|c|}{ Forecasting Performance 2004-2009 } \\
\hline & 3 month & 1 year & 3 year & 5 year & 10 year & 3 month & 1 year & 3 year & 5 year & 10 year & 3 month & 1 year & 3 year & 5 year & 10 year \\
\hline \multicolumn{16}{|c|}{ Macroeconomic SDFM } \\
\hline$h=1$ & 0.170 & 0.248 & 0.280 & 0.296 & 0.308 & 0.267 & 0.286 & 0.349 & 0.339 & 0.296 & 0.337 & 0.256 & 0.300 & 0.291 & 0.303 \\
\hline$h=6$ & 0.391 & 0.649 & 0.754 & 0.779 & 0.748 & 1.066 & 1.193 & 1.094 & 0.959 & 0.679 & 1.007 & 0.954 & 0.886 & 0.824 & 0.644 \\
\hline$h=12$ & 0.664 & 0.914 & 1.033 & 1.064 & 1.023 & 1.921 & 2.052 & 1.734 & 1.463 & 1.022 & 1.418 & 1.310 & 1.244 & 1.129 & 0.863 \\
\hline$h=18$ & 0.985 & 1.084 & 1.110 & 1.132 & 1.053 & 2.541 & 2.632 & 2.154 & 1.789 & 1.248 & 1.890 & 1.789 & 1.702 & 1.514 & 1.083 \\
\hline$h=24$ & 1.405 & 1.303 & 1.149 & 1.145 & 0.998 & 2.833 & 2.899 & 2.373 & 1.986 & 1.442 & 2.597 & 2.493 & 2.263 & 1.990 & 1.462 \\
\hline \multicolumn{16}{|c|}{ SDFM } \\
\hline$h=1$ & 0.151 & 0.252 & 0.278 & 0.292 & 0.307 & 0.281 & 0.303 & 0.354 & 0.345 & 0.298 & 0.425 & 0.268 & 0.328 & 0.309 & 0.302 \\
\hline$h=6$ & 0.488 & 0.714 & 0.781 & 0.800 & 0.761 & 1.133 & 1.239 & 1.104 & 0.965 & 0.682 & 1.112 & 1.038 & 0.995 & 0.872 & 0.604 \\
\hline$h=12$ & 0.777 & 0.946 & 1.018 & 1.049 & 1.001 & 1.970 & 2.062 & 1.706 & 1.436 & 1.004 & 1.850 & 1.729 & 1.533 & 1.297 & 0.876 \\
\hline$h=18$ & 0.896 & 0.981 & 1.033 & 1.076 & 1.015 & 2.548 & 2.611 & 2.121 & 1.768 & 1.250 & 2.447 & 2.308 & 2.010 & 1.701 & 1.150 \\
\hline$h=24$ & 1.020 & 1.046 & 1.043 & 1.085 & 0.992 & 2.952 & 3.015 & 2.484 & 2.098 & 1.558 & 2.870 & 2.728 & 2.360 & 2.005 & 1.393 \\
\hline \multicolumn{16}{|l|}{ DFM } \\
\hline$h=1$ & 0.154 & 0.252 & 0.279 & 0.294 & 0.303 & 0.305 & 0.299 & 0.350 & 0.343 & 0.297 & 0.435 & 0.267 & 0.326 & 0.309 & 0.300 \\
\hline$h=6$ & 0.477 & 0.715 & 0.784 & 0.801 & 0.770 & 1.118 & 1.214 & 1.083 & 0.950 & 0.674 & 1.105 & 1.027 & 0.978 & 0.858 & 0.596 \\
\hline$h=12$ & 0.755 & 0.941 & 1.015 & 1.039 & 1.007 & 1.931 & 2.019 & 1.666 & 1.403 & 0.980 & 1.834 & 1.708 & 1.499 & 1.264 & 0.852 \\
\hline$h=18$ & 0.850 & 0.951 & 1.003 & 1.039 & 1.007 & 2.493 & 2.550 & 2.060 & 1.711 & 1.200 & 2.431 & 2.286 & 1.969 & 1.660 & 1.114 \\
\hline$h=24$ & 0.942 & 0.975 & 0.968 & 1.004 & 0.952 & 2.877 & 2.935 & 2.402 & 2.021 & 1.486 & 2.860 & 2.712 & 2.320 & 1.960 & 1.352 \\
\hline \multicolumn{16}{|c|}{ NS-SSF } \\
\hline$h=1$ & 0.185 & 0.244 & 0.279 & 0.289 & 0.275 & 0.289 & 0.301 & 0.354 & 0.353 & 0.309 & 0.472 & 0.266 & 0.325 & 0.313 & 0.293 \\
\hline$h=6$ & 0.559 & 0.710 & 0.787 & 0.799 & 0.767 & 1.150 & 1.217 & 1.098 & 0.964 & 0.717 & 1.138 & 1.017 & 0.980 & 0.866 & 0.610 \\
\hline$h=12$ & 0.843 & 0.940 & 1.021 & 1.042 & 1.018 & 2.004 & 2.027 & 1.697 & 1.428 & 1.054 & 1.872 & 1.689 & 1.503 & 1.274 & 0.882 \\
\hline$h=18$ & 0.966 & 0.968 & 1.028 & 1.059 & 1.049 & 2.595 & 2.565 & 2.105 & 1.749 & 1.299 & 2.474 & 2.259 & 1.971 & 1.665 & 1.149 \\
\hline$h=24$ & 1.127 & 1.042 & 1.046 & 1.069 & 1.055 & 3.004 & 2.958 & 2.461 & 2.071 & 1.600 & 2.905 & 2.676 & 2.317 & 1.960 & 1.387 \\
\hline \multicolumn{16}{|l|}{ FSN } \\
\hline$h=1$ & 0.467 & 0.266 & 0.289 & 0.305 & 0.284 & 0.319 & 0.287 & 0.334 & 0.334 & 0.299 & 0.341 & 0.245 & 0.323 & 0.294 & 0.300 \\
\hline$h=6$ & 0.730 & 0.782 & 0.867 & 0.903 & 0.844 & 1.094 & 1.169 & 1.034 & 0.932 & 0.688 & 1.031 & 0.978 & 0.952 & 0.830 & 0.632 \\
\hline$h=12$ & 1.004 & 1.031 & 1.133 & 1.189 & 1.123 & 2.026 & 2.017 & 1.670 & 1.432 & 1.031 & 1.769 & 1.633 & 1.454 & 1.219 & 0.886 \\
\hline$h=18$ & 1.157 & 1.083 & 1.159 & 1.232 & 1.168 & 2.655 & 2.593 & 2.134 & 1.809 & 1.310 & 2.393 & 2.209 & 1.937 & 1.636 & 1.143 \\
\hline$h=24$ & 1.325 & 1.173 & 1.195 & 1.256 & 1.172 & 3.058 & 2.980 & 2.490 & 2.128 & 1.610 & 2.852 & 2.644 & 2.302 & 1.953 & 1.368 \\
\hline
\end{tabular}




\section{Conclusion}

We have developed a maximum likelihood procedure for incorporating many macroeconomic variables into a yield curve model with the aim of producing more precise forecasts. Our model falls within the class of dynamic factor models and we have used all recent innovations for its analysis. We have adopted cubic spline functions to introduce smoothness in factor loadings. The resulting model is referred to as the smooth dynamic factor model. From a large panel of macroeconomic series we extracted a smaller number of macroeconomic latent factors. We add these factors to our smooth dynamic factor model and obtain the macroeconomic smooth dynamic factor model. We embed the analysis within a likelihood-based framework and estimate the yield factors, macro factors and factor dynamics simultaneously.

For a newly updated time series panel of unsmoothed Fama-Bliss zero yields for U.S. treasuries, we show that adding macroeconomic information is useful for forecasting the yield curve. The estimated factor dynamics highlight interactions between the yield and macro factors. The macro factors capture information about the real economy, price indices, and labor and housing market conditions. In a comprehensive forecasting analysis, we have showed that the incorporation of macro information can lead to a lower root mean square forecast error when compared to existing yield curve models.

\section{References}

Ang, A. and M. Piazzesi (2003). A no-arbitrage vector autoregression of term structure dynamics with macroeconomic and latent variables. J. Monetary Economics 50, 74587.

Bai, J. (2003). Inferential theory for factor models of large dimensions. Econometrica 71, $135-72$.

Bliss, R. (1997). Movements in the term structure of interest rates. Federal Reserve Bank of Atlanta Economic Review 82, 16-33.

Bowsher, C. and R. Meeks (2008). The dynamics of economic functions: modeling and forecasting the yield curve. J. American Statistical Association 103, 1419-37.

Box, G. E. P., G. M. Jenkins, and G. C. Reinsel (1994). Time Series Analysis, Forecasting and Control (3rd ed.). San Francisco: Holden-Day.

Christensen, J., F. Diebold, and S. Rudebusch (2010). The affine arbitrage-free class of Nelson-Siegel term structure models. J. Econometrics (forthcoming). 
Clements, M. P. and D. Hendry (1998). Forecasting Economic Time Series. Cambridge: Cambridge University Press.

Connor, G. and R. A. Korajczyk (1993). A test for the number of factors in an approximate factor model. Journal of Finance 48(4), 1263-91.

De Pooter, M. (2007). Examining the Nelson-Siegel class of term structure models. Tinbergen Institute Discussion Paper.

Diebold, F. and C. Li (2006). Forecasting the term structure of government bond yields. J. Econometrics 130, 337-64.

Diebold, F., S. Rudebusch, and S. Aruoba (2006). The macroeconomy and the yield curve. J. Econometrics 131, 309-38.

Doz, C., D. Giannone, and L. Reichlin (2010). A quasi maximum likelihood approach for large approximate dynamic factor models. Rev. Economics and Statistics, forthcoming.

Durbin, J. and S. J. Koopman (2001). Time Series Analysis by State Space Methods. Oxford: Oxford University Press.

Engle, R. F. and M. W. Watson (1981). A one-factor multivariate time series model of metropolitan wage rates. J. American Statistical Association 76, 774-81.

Exterkate, P., D. Van Dijk, C. Heij, and P. Groenen (2010). Forecasting the yield curve in a data-rich environment using the factor-augmented Nelson-Siegel model. Econometric Institute Report.

Fama, E. F. and R. R. Bliss (1987). The information in long-maturity forward rates. American Economic Review 77, 680-92.

Forni, M., M. Hallin, M. Lippi, and L. Reichlin (2000). The generalized dynamic factor model: Identification and estimation. Rev. Economics and Statistics 82, 540-54.

Geweke, J. (1977). The dynamic factor analysis of economic time series. In D. J. Aigner and A. S. Goldberger (Eds.), Latent variables in socio-economic models. Amsterdam: North-Holland.

Geweke, J. and K. J. Singleton (1981). Maximum likelihood conrmatory factor analysis of economic time series. International Economic Review 22, 37-54.

Gregory, A., A. Head, and J. Raynauld (1997). Measuring world business cycles. International Economic Review 38, 677-701.

Harvey, A. C. and S. J. Koopman (1993). Forecasting hourly electricity demand using time-varying splines. J. American Statistical Association 88, 1228-36. 
Jungbacker, B. and S. J. Koopman (2008). Likelihood-based analysis for dynamic factor models. Tinbergen Institute Discussion Paper.

Jungbacker, B., S. J. Koopman, and M. van der Wel (2010). Smooth dynamic factor analysis with an application to the U.S. term structure of interest rates. Tinbergen Institute Discussion Paper.

Koopman, S. J. and J. Durbin (2000). Fast filtering and smoothing for multivariate state space models. J. Time Series Analysis 21, 281-96.

Koopman, S. J., M. Mallee, and M. Van der Wel (2010). Analyzing the term structure of interest rates using the dynamic Nelson-Siegel model with time-varying parameters. $J$. Business and Economic Statist. 28, 329-343.

Koopman, S. J. and N. Shephard (1992). Exact score for time series models in state space form. Biometrika 79, 823-6.

Litterman, R. and J. Scheinkman (1991). Common factors affecting bond returns. Journal of Fixed Income 1(1), 54-61.

Moench, E. (2008). Forecasting the yield curve in a data-rich environment: A no-arbitrage factor-augmented VAR approach. J. Econometrics 146, 26-43.

Monahan, J. F. (2001). Numerical methods of statistics. Cambridge: Cambridge University Press.

Nelson, C. and A. Siegel (1987). Parsimonious modelling of yield curves. Journal of Business 60-4, 473-89.

Nocedal, J. and S. J. Wright (1999). Numerical Optimization. New York: Springer Verlag.

Poirier, D. J. (1976). The Econometrics of Structural Change: with Special Emphasis on Spline Functions. Amsterdam: North-Holland.

Quah, D. and T. J. Sargent (1993). A dynamic index model for large cross sections. In J. H. Stock and M. Watson (Eds.), Business cycles, indicators and forecasting, pp. 285-306. Chicago: University of Chicago Press.

Sargent, T. J. and C. A. Sims (1977). Business cycle modeling without pretending to have too much a priori economic theory. In C. A. Sims et al. (Eds.), New methods in business cycle research. Minneapolis: Federal Reserve Bank of Minneapolis.

Stock, J. H. and M. Watson (2002). Macroeconomic forecasting using diffusion indexes. J. Business and Economic Statist. 20, 147-62. 
Stock, J. H. and M. Watson (2005). Implications of dynamic factor models for var analysis. Discussion paper.

Watson, M. W. and R. F. Engle (1983). Alternative algorithms for the estimation of dynamic factor, MIMIC and varying coefficient regression. J. Econometrics 23, 385400. 